\title{
Imaging characteristics and growth of subependymal giant cell astrocytomas
}

\author{
Michelle J. Clarke, M.D., Andrew B. Foy, M.D., Nicholas Wetjen, M.D., and Corey \\ RAFFel, M.D., Ph.D.
}

Department of Neurologic Surgery, Mayo Clinic, Rochester, Minnesota

\begin{abstract}
Object. Subependymal giant cell astrocytomas (SEGAs) are a common manifestation of tuberous sclerosis (TS). These evolving tumors have a propensity to cause obstructive hydrocephalus, usually due to obstruction at the level of the foramen of Monro. Differentiating SEGAs from subependymal nodules (SENs) before obstruction occurs may improve the morbidity associated with these tumors. In this study the authors' aim was to determine imaging characteristics of proven tumors in a single-center pediatric population.

Methods. The authors retrospectively reviewed all records and images obtained in patients with TS in whom results of biopsy sampling had proven that their tumors were SEGAs. Time to presentation, signs and symptoms at presentation, and imaging characteristics of the evolving tumors were noted. Twelve patients with 14 SEGAs proven by the results of biopsy sampling were reviewed. Resection was recommended for symptomatic and neuroimaging evidence of hydrocephalus (41\%), tumor growth without evidence of hydrocephalus (33\%), and for poorly controlled seizures $(25 \%)$. The mean diameter of the tumors at the time of resection was $1.9 \mathrm{~cm}$ (range $0.3-4 \mathrm{~cm}$ ), and no tumor recurred. Because of the pathological and radiographic continuum of SENs and SEGAs, it remains difficult to predict whether and when a given lesion will progress. Tumor growth and contrast enhancement are the most common signs of progression on neuroimages, and may be seen prior to the development of obstructive hydrocephalus.

Conclusions. Patients with SENs and SEGAs should undergo follow-up neuroimaging at yearly intervals, and if lesions show signs of progression (contrast enhancement or growth), these intervals should be shortened and consideration given to early resection.
\end{abstract}

KEY WORDS • subependymal giant cell astrocytoma $・$ subependymal nodule $・$ resection

Tuberous sclerosis is an autosomal-dominant, multisystem, neurocutaneous syndrome classically characterized by the Vogt clinical triad of seizures, mental retardation, and facial adenoma sebaceum. ${ }^{15}$ Benign tumors occur in various organs, including the heart, kidneys, and brain. In the brain, the lesions typically manifest as cortical and subependymal hamartomas or tubers; these are areas of giant neurons, astrocytes, and areas of gliosis. ${ }^{3}$

A common manifestation of TS is the SEGA. Occurring in 5 to $10 \%$ of patients with TS, ${ }^{13}$ usually in the second decade of life, ${ }^{14}$ these lesions have been theorized to arise from subcortical tubers. Although histologically benign, SEGAs commonly arise in the lateral ventricles near the foramen of Monro and can result in obstructive hydrocephalus. Historically, patients often presented with symptoms of increased intracranial pressure,,$^{11,12}$ and the death rate after surgery in the acute phase approached $10 \%$. More recently, tumors have been resected in the nonacute phase to improve prognosis. ${ }^{10}$

Abbreviations used in this paper: $\mathrm{CT}=$ computerized tomography; MR = magnetic resonance; SEGA = subependymal giant cell astrocytoma; $\mathrm{SEN}=$ subependymal nodule; $\mathrm{TS}$ = tuberous sclerosis.

\section{CLINICAL MATERIAL AND METHODS}

To obtain a list of all patients with TS who had tumors identified as SEGAs based on biopsy sampling, the Mayo Clinic medical and surgical database was queried. Between 1984 and 2004, 456 patients with TS were identified. Of these patients, an unspecified astrocytoma was diagnosed in 35 , and of this subgroup 12 had tumors identified as SEGAs based on results of biopsy sampling.

\section{Patient Population}

In the 12 patients identified, there were 14 proven SEGAs based on results of biopsy sampling. Of the 12 individuals, six were male and six were female. The mean age at diagnosis of TS was 51 months (range 1-180 months). Workup for TS was initiated due to seizures in nine patients (75\%), family history in two (16.7\%), skin lesions in one, and cardiac arrhythmia in one (8\%). One patient who had seizures also had a family history of TS, and so is counted twice. Patients with SEGAs were identified at a mean age of 90 months (range 3-180 months).

We retrospectively reviewed each of these 12 cases. The 
presenting symptoms and date of diagnosis of TS and SEGA were noted, along with the presenting signs and symptoms resulting in resection. Imaging studies (both MR and $\mathrm{CT}$ ) were reviewed by a staff radiologist, and tumor size, growth, location, and enhancement were noted. The surgical procedure and complications were noted.

\section{RESULTS}

The 12 patients with TS underwent a total of 13 procedures for resection of 14 separate SEGAs by one of four staff surgeons. Resection was recommended for symptomatic and neuroimaging evidence of hydrocephalus in nine (75\%) of 12 patients; in five (56\%) there was evidence of tumor growth, and in four (44\%) there was no evidence of growth on follow-up scans. In seven patients $(58 \%)$ resected tumors had grown since the previous neuroimage was obtained; five (71\%) had evidence of hydrocephalus, and two $(28 \%)$ had no evidence of this disorder. In one $(14 \%)$ of these seven patients a large tumor was removed after its discovery, and no additional follow-up images were obtained.

All 12 patients underwent either CT scanning (five) or MR imaging (seven) before surgery. The mean diameter of the tumors at the time of resection was $1.9 \mathrm{~cm}$ (range $0.3-4$ $\mathrm{cm}$ ). All lesions arose from the foramen of Monro. On CT scans the tumors appeared well circumscribed, isointense compared with cortex, and markedly enhanced after administration of contrast materials. Many had evidence of calcification at the periphery of the lesion. On MR imaging there was a high-intensity $T_{2}$ signal and a low-intensity $T_{1}$ signal within the tumors. There was no evidence of recent or remote hemorrhage on the neuroimages we reviewed. Tumors enhanced homogeneously after the administration of $\mathrm{Gd}$ contrast material. There was no evidence of surrounding edema.

Resection in these patients was performed at a mean age of 129 months (range 41-188 months); a mean of 38 months (range 0-142 months) elapsed between the initial diagnosis of presumed SEGA on neuroimaging and the surgery. All patients underwent craniotomy, with stereotactic guidance in five (42\%). Two patients $(16.7 \%)$ experienced postoperative weakness, which resolved completely. One patient $(8 \%)$ had a postoperative wound infection that required two additional procedures for debridement and cranioplasty. One patient (8\%) required a second craniotomy for resection of a contralateral tumor that had increased in size on postoperative imaging. No tumors recurred during the follow-up period.

\section{DISCUSSION}

Tuberous sclerosis is an autosomal-dominant neurocutaneous disease that originates during fetal development, and it gives rise to hamartomas in multiple organ systems. In the brain, four major pathological features have been identified, as follows: 1) cortical and subcortical tubers; 2) white matter disease; 3) SENs; and 4) SEGAs.

Of patients with TS, 88 to $95 \%$ have radiographic evidence of SENs, and in 2 to $14 \%$ SEGAs are diagnosed. ${ }^{11}$ Overall, SEGAs are benign, slow-growing tumors; however, their propensity to arise near the foramen of Monro may result in life-threatening hydrocephalus. In their extensive review of 216 living patients with TS complex, Shepard and colleagues ${ }^{11,12}$ found that SEGAs occurred in only 21 patients (10\% of the study population). Nevertheless, 10 of these patients died, six of them as a direct result of tumor growth (five with acute obstructive hydrocephalus and one with intratumoral hemorrhage, a rare occurrence), and four as a result of tumor treatment. Death due to brain tumor was most common in the 10- to 19-year-old age group. Although seizures form one of the three components of the Vogt triad and status epilepticus is a major cause of morbidity in this population, the incidence of seizures is not increased in patients with SEGA. ${ }^{9}$ This is understandable because cortical tubers are the most likely seizure foci in this population, not SEGAs or SENs. Thus, SEGAs are not likely to be an independent risk factor for seizure-induced deaths.

The rate of morbidity and mortality caused by SEGAinduced hydrocephalus is a common theme in the literature. Despite this, SEGAs are often not resected if "asymptomatic"; that is, if they do not appear to be the cause of hydrocephalus on neuroimaging. Recently, however, this idea has been changing.

Nabbout, et al., ${ }^{9}$ suggest that operating earlier on highrisk lesions may reduce the risk of morbidity and death. In their series, the first devoted to pediatric patients, these authors found that $40 \%$ of patients with TS had lesions near the foramen of Monro, and thus would be at risk for hydrocephalus if the tumors evolved or enlarged. Although theoretically identification of lesions that have a high risk of evolving into an SEGA (with the potential to cause acute hydrocephalus) is admirable, in practice the distinction is difficult to make.

Immunohistochemical studies support the theory that the cerebral manifestations of TS are due to divergent glioneuronal differentiation resulting in giant cells of diverse types. These studies indirectly support the theory that the lesions may result from a migrational abnormality. ${ }^{6}$ Neuroimaging studies also point to similarities between the four pathological features. All four lesions enhance to varying degrees with addition of contrast agents, indicating blood-brain barrier disruption. Braffman and colleagues ${ }^{2}$ theorize that imaging similarities relate to pathological similarities between the lesions. This is further illustrated by evidence that SENs may be seen to develop into SEGAs on serial imaging. 5,8 These imaging and pathological similarities make differentiating between the SENs and SEGAs much more difficult for the clinician.

In a small case series and literature review, Moran and $\mathrm{O}^{\prime} \mathrm{Keeffe}^{7}$ distinguished SEGAs from SENs identified on CT scans based on location and uniform enhancing characteristics. Braffman, et al., ${ }^{2}$ define the SEN as a small, nonobstructing, subependymal nodular lesion in any ventricle, whereas probable SEGAs were moderate- to large-sized subependymal or intraventricular masses that are located near the foramen of Monro and demonstrate serial growth. Braffman and colleagues also noted that SEGAs enhance more constantly than SENs with Gd administration. Unfortunately, these definitions point only to two major features; SEGAs are more likely to grow and are usually located near the foramen of Monro, a combination with obvious risks for obstructive hydrocephalus.

Nabbout, et al., ${ }^{9}$ in their retrospective cohort study, re- 
viewed a group of patients with TS in whom SENs were identified near the foramen of Monro and found that lesions that were not calcified enhanced after Gd administration and evolved into SEGAs at a higher rate, especially in patients with a family history of TS. Although helpful, these guidelines are not sufficient to stratify a SEN or SEGA as a high or low risk for obstructive complications.

One of the most important pieces of data collected recently is the timeframe of evolution of the lesions. Braffman and associates ${ }^{2}$ noted a patient in whom a lesion progressed from a small SEN to an obstructive SEGA within 18 months. Thus, these authors recommend that serial scans be performed annually or biannually to identify progression before obstruction has occurred.

Early detection of progressing lesions is only important, however, if it results in practice change. In 1982, Eisenberg $^{4}$ recommended surgery only in patients with symptomatic disease. As expected, the most common reason for surgery was elevated intracranial pressure. Nevertheless, numerous publications have espoused earlier surgery to avoid the sequelae of hydrocephalus. ${ }^{1,2,8}$ Surgical treatment has ranged from cerebrospinal fluid-diverting procedures (which one of our patients underwent before resection) to removal of the lesion. Complete resection yields a low rate of tumor recurrence. This is evidenced by our study population, in which no tumor recurred after gross-total resection. One patient did undergo a second craniotomy for residual tumor after undergoing the initial resection at another institution.

As evidenced by our data set, we too agree that early surgery or close follow-up review should be advocated. Four of the 12 patients in our study underwent resection of a lesion due to its size (one patient) or growth (three patients), without evidence of obstructive hydrocephalus. Unfortunately, monitoring the tumors for growth may not provide the answer; three of the patients underwent surgery after hydrocephalus developed less than 1 year after the SEGA diagnosis. Preoperative imaging demonstrating tumor growth and a follow-up MR image obtained after resection are shown in Fig. 1.

\section{CONCLUSIONS}

Due to the propensity of SENs to progress to SEGAs, we suggest close follow-up evaluation for all known lesions, especially those located near the foramen of Monro. Because there is evidence that these lesions can evolve to obstruct the foramen in as little as 18 months, follow-up imaging performed at yearly intervals is prudent. Evidence of progression (contrast enhancement or increased growth) would lead to closer observation and consideration of resection. We advocate that high-risk lesions be removed when possible before symptoms of obstructive disease arise.

\section{References}

1. Boesel CP, Paulson GW, Kosnik EJ, et al: Brain hamartomas and tumors associated with tuberous sclerosis. Neurosurgery 5:410-417, 1979

2. Braffman BH, Bilaniuk LT, Naidich TP, et al: MR imaging of tuberous sclerosis: pathogenesis of this phakomatosis, use of gadopentetate dimeglumine, and literature review. Radiology 183:227-238, 1992

3. Doran SE, Thorell WE: Genetic and hereditary syndromes associated with brain tumors, in Winn R (ed): Youmans Neurological Surgery, ed 5. Philadelphia: WB Saunders, 2004, pp 811-815

4. Eisenberg HM: Supratentorial astrocytoma, in Pediatric Neurosurgery: Surgery of the Developing Nervous System. New York: Grune and Stratton, 1982, pp 429-432

5. Fujiwara S, Takaki T, Hikita T, et al: Subependymal giant-cell astrocytoma associated with tuberous sclerosis. Do subependymal nodules grow? Childs Nerv Syst 5:43-44, 1989

6. Hirose T, Scheithauer BW, Lopes MB, et al. Tuber and subep-
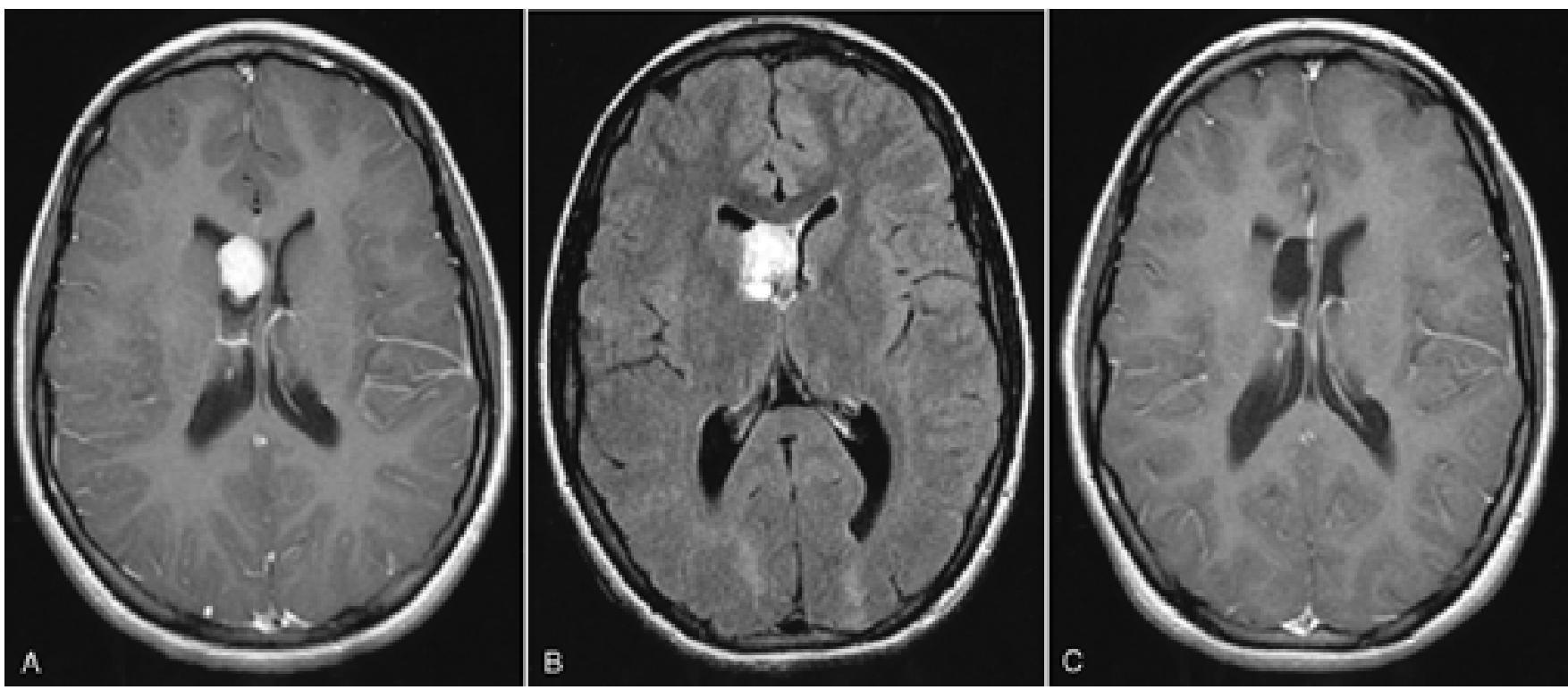

Fig. 1. Neuroimages revealing a SEGA near the right foramen of Monro. A: Preoperative image obtained 14 months before resection. B: Preoperative image obtained 1 month before resection, demonstrating tumor enlargement. C: Postoperative image showing absence of residual tumor. 
endymal giant cell astrocytoma associated with tuberous sclerosis: an immunohistochemical, ultrastructural, and immunoelectron and microscopic study. Acta Neuropathol (Berl) 90: 387-399, 1995

7. Moran V, O'Keeffe F: Giant cell astrocytoma in tuberous sclerosis: computed tomographic findings. Clin Radiol 37:543-545, 1986

8. Morimoto K, Mogami H: Sequential CT study of subependymal giant cell astrocytomas associated with tuberous sclerosis. Case report. J Neurosurg 65:874-877, 1986

9. Nabbout R, Santos M, Rolland Y, et al: Early diagnosis of subependymal giant cell astrocytomas in children with tuberous sclerosis. J Neurol Neurosurg Psychiatry 66:370-375, 1999

10. Nagib MG, Haines SJ, Erickson DL, et al: Tuberous sclerosis: a review for the neurosurgeon. Neurosurgery 14:93-98, 1984

11. Shepard CW, Gomez MR: Mortality in the Mayo Clinic tuberous sclerois complex study. Ann N Y Acad Sci 615:375-377, 1991

12. Shepard CW, Gomez MR, Lie JT, et al: Causes of death in pa- tients with tuberous sclerosis. Mayo Clin Proc 66:792-796, 1991

13. Shepard CW, Scheithauer B, Gomez MR: Brain tumors in tuberous sclerosis. A clinicopathologic study of the Mayo clinic experience. Ann N Y Acad Sci 615:378-379, 1991

14. Shepherd C, Scheithauer B, Gomez M, et al: Subependymal giant cell astrocytoma: A clinical, pathological, and flow cytometric study. Neurosurgery 28:864-868, 1991

15. Vogt H: Sur Pathologie und pathologischen Anatomie der verschieden Idiotieforem. Monatsschr Psychiat Neurol 24: 106-150, 1908

Manuscript received November 12, 2005.

Accepted in final form December 9, 2005.

Address reprint requests to: Michelle Clarke, M.D., Department of Neurologic Surgery, Mayo Clinic, 200 First Street South West, Rochester, Minnesota 55905. email: clarke.michelle@mayo.edu. 